\title{
Use of transcriptional slippage for diverse gene expression*
}

\begin{abstract}
Department of Microbiology, Faculty of Biology, University of Gdansk, Gdańsk, Poland
We present here an alternative for two-promoter systems ensuring highly diverse expression of several genes from a single promoter. This approach assumes an introduction of a deletion mutation into an A/T homopolymeric run in a gene's proximal part, and employs the transcriptional slippage mechanism for insertion-dependent reinstatement of the proper reading frame by the T7 RNA polymerase.
\end{abstract}

Dawid Koscielniak, Ewelina Sobisz, Ewa Wons and Marian Sektas ${ }^{\square}$ 2015).
Keywords: GFP reporter, expression system, InDel error, M2.Mboll methyltransferase, T7 bacteriophage RNA polymerase, transcriptional slippage

Received: 07 June, 2021; revised: 28 July, 2021; accepted: 29 July, 2021; available on-line: 26 August, 2021

Ðe-mail: marian.sektas@ug.edu.pl

*This paper is dedicated to Professor Wacław Tadeusz Szybalski on the 100th anniversary of his birth

Abbreviations: FS, frameshifted variants; InDel, either insertion or deletion of a nucleotide; WT, wild-type inframe variants

During the transcription process, in many regions of long homopolymeric poly(A) or poly(T) sequences a phenomenon of insertion or deletion of one or more nucleotides might occur (transcriptional slippage, Atkins et al., 2016). Programmed transcriptional slippage always occurs in well-defined homopolymeric sequences of some genes and contributes to the formation of an additional alternative protein that is essential for the cell function. This happens with high efficiency, and has a specific one-directional effect (either insertion or deletion [InDel] of a nucleotide), which is often ensured by the presence of additional second-order DNA structures that are necessary to make this process more likely (Penno et al., 2015). In case of some groups of genes (e.g. IS transposases) slippage is absolutely necessary for formation of the correct and functional protein (Baranov et al., 2005; Liu et al., 2018). The nature of the non-programmed transcriptional slippage is different. It is mainly related to the intrinsic property of the RNA polymerase (RNAP), which under certain conditions (long A/T homopolymeric sequences) may introduce random and two-directional InDel errors, contributing to an increase in mRNA polymorphism of a given gene (Larsen et al., 2000; Tamas et al., 2008; Wagner et al., 1990; Wons et al., 2015), with changes in the primary reading frame (InDel frameshift). This leads to production of altered forms of proteins (Schwarz et al., 2021; Wons et al., 2015; RockahShmuel et al., 2013). Such a mechanism turns out to be beneficial in case of genes with a single InDel mutation, as it can "repair" the mRNA sequence (including reinstatement of the correct reading frame) while keeping the gene's DNA sequence mutated (Gordon et al., 2013; Koscielniak et al., 2018; Rockah-Shmuel et al., 2013; Ta- mas et al., 2008; Wernegreen et al., 2010; Wons et al.,

Many methods based on various protein overproduction systems are focused on efficient expression of a desired gene that assumes fidelity and reproducibility of this process. In the case of co-expression, employment of two different expression systems controlled independently is most widely used, leading to varied coexpressed protein proportions. Alternatively, usage of the same type of a promoter results in a balanced and equal level of both genes' expression (Novy et al., 2002). Here, we propose a novel approach to achieve a highly different expression level of two or more genes from a single controllable promoter. The method presented takes advantage of a very effective and commonly used bacteriophage T7 expression system. A reduction in protein production is achieved by introduction of a single nucleotide deletion (frameshift mutation) within the polyA/T sequence of the proximal part of a given gene. Reinstatement of the proper reading frame is insertion-dependent and employs transcriptional slippage mechanism of the T7 RNAP. This enzyme is highly capable of transcriptional slippage on such sequences, especially by nucleotide insertion (Koscielniak et al., 2018; Wons et al., 2015; Wons et al., 2018), and what is important, in contrast to the host polymerase, T7 RNAP generated expression manifests its intrinsic resistance to transcriptional polarity in the absence of T7-specific terminators (ChevrierMiller et al., 1990). Feasibility of the proposed approach is demonstrated here with combination of tandem genes: mboIIM2 and gfp (transcriptional fusion, each gene possesses its own Shine-Dalgarno sequence), encoding the DNA methyltransferase M2.MboII and GFP proteins, respectively, both as inframe (WT) and frameshifted (FS) variants (Fig. 1). The four combinations of tandem genes were introduced into the pET24a plasmid vector (Novagene) and were constructed as follows: WT or FS gfp gens were subcloned from pBADmingfp $\mathrm{A}_{6} 0$ and pBADmingfp $\mathrm{A}_{5}-1$ as EcoRI-digested DNA fragments (Wons et al., 2018) and inserted into the EcoRI site of pETmboIIMB.3 (carrying mboIIM2 W'T gene, Furmanek-Blaszk et al., 2009) or pETmboIIM2FS vectors (site-specific mutagenized variant of mboIIM2 with deletion of the 18th thymine residue, using the PfuPlus DNA polymerase in a PCR reaction - Eurx-Gdansk, Poland) (Fig. 1). All plasmids were introduced into Escherichia coli ER2566 strain which hosts the T7 RNA polymerase (NEB Ipswich, USA). After $1 \mathrm{~h}$ induction with $1 \mathrm{mM}$ IPTG, the effects of diverse levels of expression were assayed both, qualitatively and quantitatively (at least in the case of GFP protein) (Fig. 2). For GFP analysis, measurement of cell fluorescence and immunodetection of the protein by western blotting were used (Wons et al., 2018). For M2.MboII, an in vivo methylation test was utilized (DNA protection against cognate $\mathrm{MboII}$ endonuclease cleavage) 


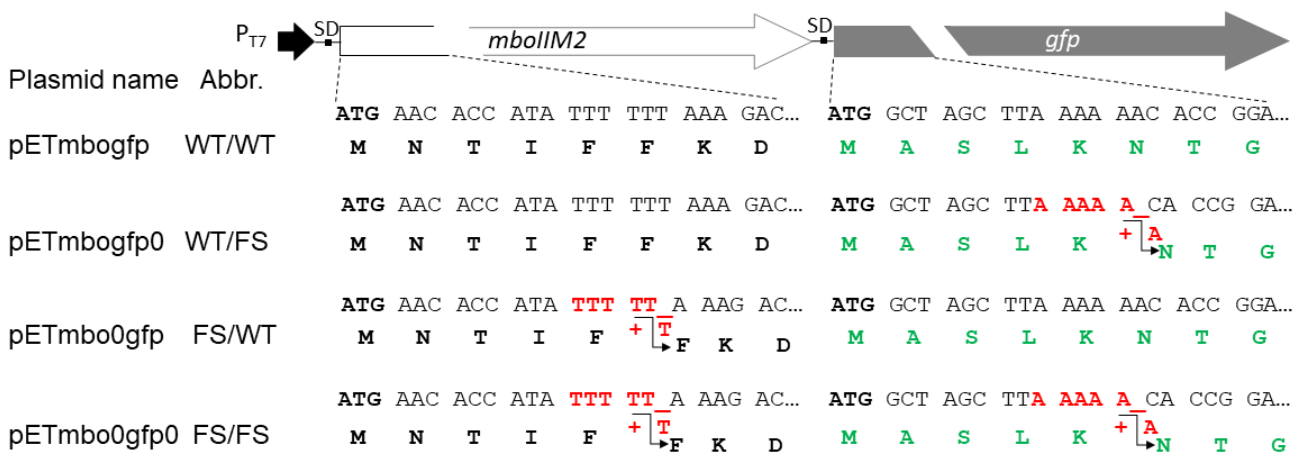

Figure 1. Details of the relevant sequence of the mbollM2 and gfp transcriptional fusion genes, in four tandem combinations including the wild-type (WT) and frameshifted variants (FS, single nucleotide deletion).

The plasmid names, abbreviations of the gene combinations, SD (Shine-Dalgarno) sequence and amino acids residues are shown. The location of the A/T homopolymeric runs (bold, in red) deletion sites are marked as a line in red. The likely site of nucleotide insertion (either $+\mathrm{A}$ or $+\mathrm{T}$ ) in homopolymer runs after slippage is marked by an arrow.
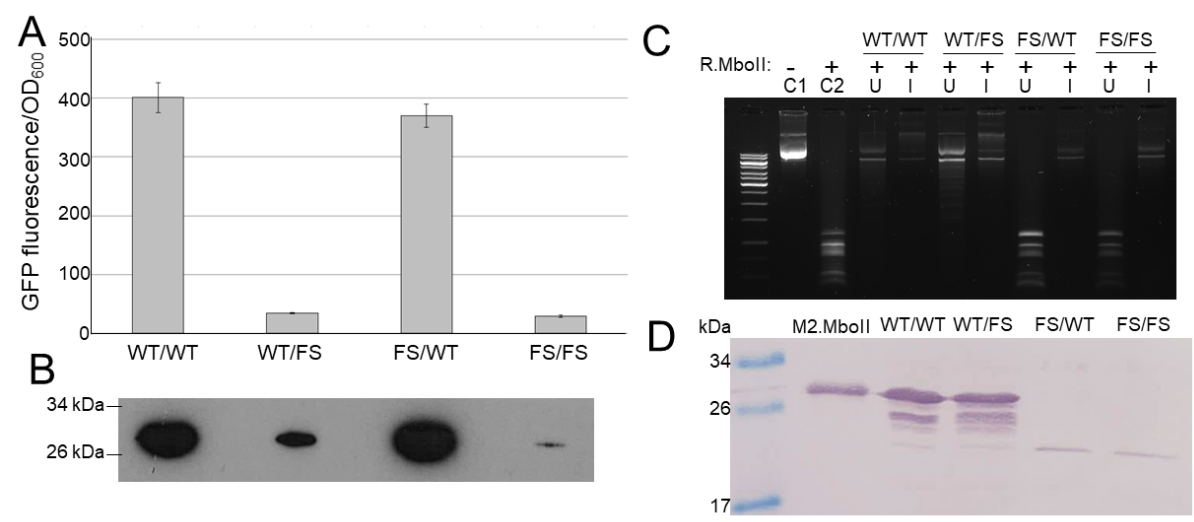

Figure 2. Expression levels of the four combinations of mbollM2 and gfp gene variants.

(A) Relative GFP activity shown in fluorescence units normalized to $O D_{600}$ of the bacterial culture. Error bars represent standard deviation from at least five independent experiments. (B) Expression levels of the corresponding gfp constructs by western blotting and immunodetection of GFP. (C) Relative level of plasmid DNA methylation by M2.Mboll challenged by R.Mboll endonuclease digestion. (-/+) denotes untreated and R.Mboll digested DNAs, respectively: C1 - untreated pETmbo0gfp0; $C 2$ - digested unmethylated pETgfp; U plasmids from uninduced bacteria; I - plasmids from induced bacteria (1 mM IPTG by $1 \mathrm{~h}$ ). (D) Expression levels of the corresponding mbollM2 constructs assessed by western blotting and immunodetection of M2.Mboll after $1 \mathrm{~h}$ induction with $1 \mathrm{mM}$ IPTG.

and immunodetection was determined by western blotting (Wons et al., 2015). Quantification of GFP fluorescence of a $400 \mu \mathrm{l}$ cell culture sample (Varioskan Flash Spectral Scanning Multimode Reader spectrophotometer - Thermo Scientific, with excitation and emission wavelengths of 488 and $510 \mathrm{~nm}$, respectively) (Fig. 2A) is proportional to immunodetected GFP products (mouse monoclonal anti-GFP (B-2) antibodies - Santa Cruz Biotechnology, Fig. 2B) (Wons et al., 2018). As can be seen, low level of gfp expression was obtained even when FS $g / p$ variant was employed, with no effect of the upstream mboIIM2 gene's status. Similarly, M2.MboII production was immunodetected (Fig. 2D, rabbit polyclonal antiM2.MboII) and the enzyme's activity was determined as follows: plasmid DNA with $18 \mathrm{MboII}$ sites was isolated from bacterial cells carrying appropriate expression plasmids and then treated with cognate MboII restriction endonuclease (Wons et al., 2015) (Fig. 2C). All plasmids from induced cells were protected against endonuclease cleavage, even though M2.MboII was not detected by immunodetection in the FS mboIIM2 samples. Again, this demonstrates that a low level of WT mboIIM2 expression took place even in the FS variant and this was not affected by the gfp gene's status.
In summary, we demonstrate here that transcriptional slippage is a suitable tool for restoring the WT reading frame in a gene containing a frameshift mutation and obtaining a low level of its expression, while the tandem WT/WT variants retain a high level of expression. This system can be used wherever the production of a valuable molecular poison can only take place in the presence of an antidote that must be removed during purification, which always increases the steps in detection and control procedures.

\section{REFERENCES}

Atkins JF, Loughran G, Bhatt PR, Firth AE, Baranov PV (2016) Ribosomal frameshifting and transcriptional slippage: From genetic steganography and cryptography to adventitious use. Nucleic Acids Res 44: 7007-7078. https://doi.org/10.1093/nar/gkw530

Baranov PV, Hammer AW, Zhou J, Gesteland RF, Atkins JF (2005) Transcriptional slippage in bacteria: distribution in sequenced genomes and utilization in IS element gene expression. Genome Biol 6: R25. https://doi.org/101186/gb-2005-6-3-r25

Chevrier-Miller M, Jacques N, Raibaud O, Dreyfus M (1990) Transcription of single-copy hybrid lacZ genes by T7 RNA polymerase in Escherichia coli: mRNA synthesis and degradation can be uncoupled from translation. Nucleic Acids Res 18: 5787-5792. https://doi. org/10.1093/nar/18.19.5787 
Furmanek-Blaszk B, Boratynski R, Zolcinska N, Sektas M (2009) M1.MboII and M2.MboII type IIS methyltransferases: different specificities, the same target. Microbiology 155: 1111-1121. https://doi. org/10.1099/mic.0.025023-0

Gordon AJ, Satory D, Halliday JA, Herman C (2013) Heritable change caused by transient transcription errors. PLOS Genet 9: e1003595. https://doi.org/10.1371/journal.pgen.1003595

Koscielniak D, Wons E, Wilkowska K, Sektas M (2018) Non-programmed transcriptional frameshifting is common and highly RNA polymerase type-dependent. Microb Cell Fact 17: 184. https://doi. org/10.1186/s12934-018-1034-4

Larsen B, Wills NM, Nelson C, Atkins JF, Gesteland RF (2000) Nonlinearity in genetic decoding: homologous DNA replicase genes use alternatives of transcriptional slippage or translational frameshifting. Proc Natl Acad Sci USA 97: 1683-1688. https://doi.org/10.1073/ pnas.97.4.1683

Novy R, Yaeger K, Held D, Mierendorf R (2002) Coexpression of multiple target proteins in E. coli. inNovations 15: 2-6

Penno C, Sharma V, Coakley A, O’Connell Motherway M, van Sinderen D, Lubkowska L, Kireeva ML, Kashlev M, Baranov PV, Atkins JF (2015) Productive mRNA stem loop-mediated transcriptional slippage: crucial features in common with intrinsic terminators. Proc Natl Acad Sci USA 112: E1984-E1993. https://doi. org/10.1073/pnas.1418384112

Rockah-Shmuel L, Toth-Petroczy A, Sela A, Wurtzel O, Sorek R, Tawfik DS (2013) Correlated occurrence and bypass of frame-shifting insertion-deletions (InDels) to give functional proteins. PLoS Genet 9: e1003882. https://doi.org/10.1371/journal.pgen.1003882
Schwarz MGA, Antunes D, Correa PR, da Silva-Goncalves AJ, Malaga W, Caffarena ER, Guilhot C, Mendonca-Lima L (2021) Mycobacterium tuberculosis and $M$. bovis BCG Moreau fumarate reductase operons produce different polypeptides that may be related to non-cannonical functions. Front Microbiol 11: 624121. https://doi.org/10.3389/ fmicb.2020.624121

Tamas I, Wernegreen JJ, Nystedt B, Kauppinen SN, Darby AC, Gomez-Valero L, Lundin D, Poole AM, Andersson SGE (2008) Endosymbiont gene functions impaired and rescued by polymerase infidelity at poly(A) tracts. Proc Natl Acad Sci USA 105: 14934 14939. https://doi.org/10.1073/pnas.0806554105

Wagner LA, Weiss RB, Driscoll R, Dunn DS, Gesteland RF (1990) Transcriptional slippage occurs during elongation at runs of adenine or thymine in Escherichia coli. Nucleic Acids Res 18: 3529-3535. https://doi.org/10.1093/nar/18.12.3529

Wernegreen JJ, Kauppinen SN, Degnan PH (2010) Slip into something more functional: selection maintains ancient frameshifts in homopolymeric sequences. Mol Biol Evol 27: 833-839. https://doi. org $/ 10.1093 / \mathrm{molbev} / \mathrm{msp} 290$

Wons E, Furmanek-Blaszk B, Sektas M (2015) RNA editing by T7 RNA polymerase bypasses InDel mutations causing unexpected phenotypic changes. Nucleic Acids Res 43: 3950-3963. https://doi. org/10.1093/nar/gkv269

Wons E, Koscielniak D, Szadkowska M, Sektas M (2018) Evaluation of GFP reporter utility for analysis of transcriptional slippage during gene expression. Microb Cell Fact 17: 150. https://doi.org/10.1186/ s12934-018-0999-3 